811.131.1'367.625

811.163.41'367.625

811.131.1:811.163.41

https://doi.org/10.18485/italbg.2017.1.4

\author{
Milena Popović Pisarri*
}

Università di Belgrado

\title{
L'INFINITO PASSATO ITALIANO RETTO DA VERBI MODALI A CONTROLLO E IL SUO EQUIVALENTE IN SERBO
}

Abstract: Il presente contributo ha l'obiettivo di mettere in evidenza il fatto che in italiano, tra tutti i verbi modali a controllo (sia quelli in senso stretto che quelli in senso lato), soltanto volere e preferire (marginalmente anche desiderare e gradire) possono reggere una forma verbale indicante l'anteriorità, cioè un infinito passato. In questo caso pare però esserci una preferenza della lingua italiana per il condizionale, presente o passato, di volere e preferire. A questo proposito, abbiamo ritenuto necessario porre l'accento sul fatto che l'infinito passato italiano è commutabile con un costrutto di modo finito al congiuntivo trapassato nonostante in caso di coreferenza di soggetti in italiano valga la restrizione sull'uso del congiuntivo nelle strutture subordinate rette da un verbo che richiede il congiuntivo. Abbiamo analizzato anche l'equivalente serbo dell'infinito passato italiano - il costrutto di modo finito $d a+$ perfekt, non commutabile con un infinito. Sia nel caso dell'infinito passato italiano che nel caso del costrutto $d a+$ perfekt abbiamo riscontrato un'analogia di modi e tempi rispettivamente con il periodo ipotetico italiano contenente un condizionale nella reggente e un congiuntivo nella subordinata e con il periodo ipotetico serbo contenente un condizionale nella reggente e il costrutto $d a+$ perfekt nella subordinata.

Parole chiave: italiano, serbo, infinito passato, verbi modali, controllo, da + perfekt.

\section{INTRODUZIONE}

L'infinito italiano è un modo verbale indefinito che dispone di due tempi, il presente (parlare, uscire, svegliarsi) e il passato (avere visto, essere uscito, essersi svegliato). Sia l'infinito presente (detto anche semplice) che

\footnotetext{
*uudinama@yahoo.com
} 
l'infinito passato (detto anche composto) possono svolgere la funzione di complemento dei verbi modali. A tal proposito, va sottolineato il fatto che l'infinito presente indica la contemporaneità o la posteriorità, mentre quello passato segnala l'anteriorità rispetto al tempo del verbo reggente. Prima di passare alla descrizione dell'uso dell'infinito passato complemento dei verbi modali a controllo, è opportuno fare distinzione tra i verbi modali a controllo e i verbi modali a sollevamento.

\section{I VERBI MODALI A CONTROLLO IN ITALIANO E IN SERBO}

Per controllo ${ }^{1}$ si intende la coreferenza del soggetto non espresso di una costruzione infinitiva con il soggetto (1a) o con l'oggetto, diretto o indiretto (1b), della struttura reggente.

(1) a. Luigi ha promesso ai bambini di portarli al parco ${ }^{2}$.

b. Luigi ha chiesto ai bambini di non allontanarsi.

Per sollevamento si intende lo spostamento di un sintagma nominale (di seguito $\mathrm{SN}$ ) dalla posizione del soggetto di una costruzione subordinata di modo finito nella posizione del soggetto della struttura reggente ((2a)(2b)). Il verbo di modo finito della costruzione subordinata passa intanto alla forma infinitivale.

(2) a. Sembra che Luigi capisca tutto al volo.

b. Luigi sembra capire tutto al volo.

I verbi modali in italiano sono volere, sapere, potere, dovere, avere e solere (v. Manzini et al. 1991: 514). Di questi verbi, nella categoria dei verbi a controllo rientrano soltanto volere e sapere, mentre potere, dovere, avere e solere appartengono alla categoria dei verbi a sollevamento (v. Manzini et al. 1991: 544).

La differenza principale tra i verbi a controllo e i verbi a sollevamento sta nel fatto che i verbi a controllo assegnano un ruolo tematico al SN che occupa la posizione del loro soggetto (Anna vorrebbe sparire vs. * La valigia di Anna vorrebbe sparire). Un verbo a sollevamento invece non assegna alcun ruolo tematico al SN che si trova nella posizione del suo soggetto, per cui tutti i SN che occupano la posizione del soggetto del verbo di una subordinata retta da un verbo a sollevamento possono diventare soggetti

\footnotetext{
${ }^{1}$ Per maggiori informazioni sui verbi a controllo e a sollevamento in italiano v. Donati (2008), Graffi (1994), Manzini et al. (1991) e Salvi \& Vanelli (2004).

${ }^{2}$ Tutti gli esempi presenti in questo contributo sono stati compilati ad hoc dalla stessa autrice e poi verificati con alcuni parlanti nativi.
} 
dello stesso verbo a sollevamento (Sembra che Anna/la valigia di Anna sia sparita vs. Anna/La valigia di Anna sembra essere sparita). Nelle costruzioni costituite da un verbo a controllo e un infinito da esso retto esistono dunque due elementi cruciali, coreferenti tra loro - il soggetto non espresso dell'infinito e il soggetto o l'oggetto del verbo reggente. Nelle costruzioni costituite da un verbo a sollevamento e un infinito da esso retto c'è un solo elemento chiave - il soggetto del verbo della subordinata che si è spostato nella posizione del soggetto del verbo reggente, ragione per cui in questo caso non si parla di coreferenza (o identità) di soggetti, tipica dei verbi a controllo, ma di condivisione del soggetto (v. Moskovljević 2008: 89).

In italiano esiste un gruppo di verbi che, sia semanticamente che sintatticamente, risultano particolarmente affini ai verbi modali, soprattutto a quelli a controllo. Si tratta di verbi quali preferire, desiderare, gradire, amare, adorare, odiare, detestare ${ }^{3}$, ecc. Rientrano nella categoria dei verbi a controllo e si differenziano dai verbi modali a controllo (volere e sapere) per il solo fatto che non ammettono la ristrutturazione ${ }^{4}$, pur condividendo con essi i seguenti due tratti: 1) la reggenza diretta (senza preposizione) di un infinito e 2) la coreferenza del soggetto sottinteso dell'infinito con il soggetto del verbo reggente, tipica di tutti i verbi a controllo (Voglio farlo/Lo voglio fare vs. Preferisco farlo/* Lo preferisco fare).

Esprimendo volontà, volere si usa normalmente con un soggetto animato e regge un infinito presente, il quale instaura un rapporto di contemporaneità (o posteriorità) con il tempo della frase reggente e il cui soggetto non espresso è coreferente con il soggetto del verbo reggente ( $3 \mathrm{a})$. Come vedremo più avanti, una sola eccezione alla regola della contemporaneità/ posteriorità dell'infinito (presente) retto da volere è costituita da un infinito passato retto dal condizionale di volere $(3 \mathrm{~b})$. Volere può essere costruito anche con un $\mathrm{SN}$ in funzione di oggetto diretto (3c). Può reggere anche una costruzione di modo finito (al congiuntivo) senza coreferenza di soggetti $(3 \mathrm{~d})$ :

(3) a. Voglio parlare con te.

b. Vorrei essermi già laureata.

\footnotetext{
${ }^{3}$ Sono verbi esprimenti desiderio, gradimento, ecc.

${ }^{4}$ È considerata una costruzione ristrutturata quella in cui "verbo reggente e infinito costituiscono un complesso verbale e i complementi dell'infinito vengono trattati come complementi del complesso verbale" (Manzini et al. 1991: 513). In italiano è ammessa dai verbi modali, aspettuali e di movimento. Le proprietà sintattiche delle costruzioni ristrutturate sono: 1) i clitici si posizionano davanti al verbo reggente; 2) l'oggetto diretto dell'infinito può diventare soggetto della costruzione del si passivo; 3) l'infinito non può essere negato indipendentemente; 4) con alcuni verbi a ristrutturazione avviene il cambio dell'ausiliare (v. Manzini et al. 1991: 514-516).
} 
c. Voglio un cuscino.

d. Voglio che vengano anche loro.

Con i verbi italiani che reggono il congiuntivo, volere compreso, non è ammessa la costruzione di modo finito al congiuntivo se il soggetto della struttura subordinata è coreferente con il soggetto della struttura reggente. In questo caso è obbligatorio l'uso dell'infinito (cfr. (4a)-(4d)).

(4) a. *Voglio che (io) parli con te.

b. Voglio parlare con te.

c. *Spero che (io) vada alla sua festa.

d. Spero di andare alla sua festa.

Questa restrizione viene spiegata nel seguente modo: "Il congiuntivo fa di due frasi, la reggente e la dipendente al congiuntivo, un'area sintattica unica in cui vale la restrizione: il soggetto del congiuntivo, anche se espresso solo con la flessione verbale, entra in conflitto di referenza col soggetto (anche se sottinteso) della reggente; diventa per questo impossibile interpetare come coreferenti i due soggetti in una frase come Mario pensa che ci vada domani. Se invece si usa l'indicativo (Mario pensa che ci va domani), le frasi diventano due territori autonomi, e i soggetti possono essere coreferenti senza problemi. La restrizione può essere evitata anche in un altro modo, cioè mettendo il verbo della dipendente all'infinito: non avendo questo modo verbale una flessione personale, la frase non contiene un elemento pronominale che provochi il conflitto di referenza col pronome soggetto della reggente" (Benincà 2003: 265-266). Con i verbi che reggono il congiuntivo il "conflitto di referenza" dunque non si verifica se il verbo della subordinata si usa all'indicativo (nella maggioranza dei casi non conforme alla norma linguistica) o all'infinito. Più avanti vedremo se questa restrizione blocchi anche l'uso del congiuntivo al posto dell'infinito retto dal condizionale dei verbi modali a controllo.

Il verbo modale sapere esprime capacità, si usa normalmente con un soggetto animato e regge un infinito presente, il quale indica un rapporto di contemporaneità con il tempo della frase reggente e il cui soggetto non espresso è coreferente con il soggetto del verbo reggente (5a). Non si usa mai con un costrutto di modo finito (5b). Oltre al verbo modale sapere, c'è un altro verbo sapere, non modale, a controllo, con il significato di 'essere cosciente di'. Si usa normalmente con un soggetto animato, regge un infinito presente o passato (commutabile con un costrutto di modo finito all'indicativo) o una costruzione di modo finito senza coreferenza di soggetti (cfr. $(5 c)-(5 g))$. 
(5) a. So nuotare.

b. *So che (io) nuoti/nuoto.

c. So di avere ragione.

d. So che ho ragione.

e. So di aver fatto bene.

f. So che ho fatto bene.

g. So che abitano lontano.

In serbo, come in italiano, esiste un gruppo di verbi affini ai verbi modali (želeti 'desiderare', voleti 'amare', obožavati 'adorare', mrzeti ' 'odiare', ecc.), ai quali ci riferiremo adoperando la denominazione modalni glagoli $u$ širem smislu (verbi modali in senso lato), mentre i verbi modali hteti 'volere', umeti 'sapere', znati 'sapere', moći 'potere', morati 'dovere', smeti 'potere, osare', trebati 'dovere' e imati 'avere' verranno più precisamente denominati modalni glagoli u užem smislu (verbi modali in senso stretto). A differenza dei verbi modali in senso stretto, che possono essere a controllo ${ }^{7}$ (hteti, umeti, znati) o a sollevamento (moći, morati, smeti, trebati, imati), tutti $\mathrm{i}$ verbi modali in senso lato appartengono alla categoria dei verbi a controllo (želeti, voleti, obožavati, mrzeti, ecc.). Per una maggiore semplicità di esposizione, di seguito applicheremo queste due denominazioni anche in riferimento ai verbi modali italiani distinguendo tra $\mathrm{i}$ verbi modali in senso stretto (tra i quali volere e sapere sono verbi a controllo, mentre potere e dovere sono verbi a sollevamento) e i verbi modali in senso lato (preferire, desiderare, gradire, amare, adorare, odiare, detestare, ecc.), che risultano invece essere tutti verbi a controllo.

Considerato l'argomento principale del nostro contributo, cioè l'infinito passato italiano retto da volere e verbi affini e il suo equivalente serbo, riteniamo più opportuno rinviare al prossimo paragrafo l'analisi dell'uso dei corrispondenti verbi modali serbi che reggono l'equivalente serbo dell'infinito passato italiano - la costruzione $d a+$ perfekt (congiunzione $d a+$ perfetto composto).

${ }^{5}$ Come i corrispondenti verbi italiani, anche questi esprimono desiderio, gradimento, ecc.

${ }^{6}$ A proposito di termini come modalni glagoli u užem smislu e modalni glagoli $u$ širem smislu v. Pranjković (2013: 187-190).

${ }^{7}$ Per una più dettagliata analisi dei verbi a controllo e a sollevamento in serbo/croato v. Moskovljević (2008) e Gnjatović \& Matasović (2013). 


\section{L'INFINITO PASSATO ITALIANO E $D A+P E R F E K T$ RETTI DA VERBI MODALI A CONTROLLO}

Dato che i verbi reggenti impongono restrizioni relativamente alla scelta del tempo dell'infinito, l'infinito passato italiano può essere retto dal verbo modale a controllo volere (e non dal verbo modale a controllo sapere) in quanto volere è compatibile non solo con l'idea della contemporaneità (o della posteriorità) indicata dalla forma infinitivale semplice, ma anche con l'idea dell'anteriorità espressa dalla forma infinitivale composta (cfr. (6a)(6h)). Il verbo sapere, a differenza di volere, è compatibile solo con l'idea della contemporaneità indicata dall'infinito semplice (cfr. (6i)-(6n)).

(6) a. Voglio laurearmi.

b. ?Voglio essermi laureata.

c. Vorrei laurearmi.

d. Vorrei essermi laureata.

e. Volevo laurearmi.

f. ?Volevo essermi laureata.

g. Avrei voluto laurearmi.

h. Avrei voluto essermi laureata.

i. So cucinare.

1. * So aver cucinato.

m. Saprei cucinare (se proprio dovessi farlo).

n. *Saprei aver cucinato ${ }^{8}$.

Dagli esempi riportati sopra possiamo vedere che un infinito passato è preferibilmente retto dal verbo volere al condizionale, semplice o composto ((6d) e (6h)). Più avanti cercheremo di dare una spiegazione a questa restrizione, a nostro avviso poco o per nulla segnalata nelle grammatiche italiane destinate sia ai parlanti italiani che agli apprendenti stranieri. Nonostante non sia raro trovare esempi di uso dell'infinito passato retto da volere al condizionale nelle grammatiche della lingua italiana, non abbiamo trovato né approfondimenti al riguardo nelle stesse grammatiche né contributi scientifici relativi all'uso quasi esclusivo del condizionale di volere con le forme infinitivali composte. Esempi contenenti un infinito passato retto dal

${ }^{8}$ A differenza di (61) e (6n), entrambe agrammaticali a causa dell'impossibilità di usare il verbo modale sapere con un infinito passato, frasi come So di aver cucinato bene (perché mi hai chiesto subito la ricetta) o Saprei di aver cucinato bene (se mi chiedessi la ricetta) sono grammaticali perché il verbo (non modale) sapere con il significato di 'essere cosciente di' si usa sia con l'infinito presente che con l'infinito passato, come abbiamo già messo in evidenza in $(5 \mathrm{c})$ e $(5 \mathrm{e})$. 
presente come (6b), dall'imperfetto come (6f) o, eventualmente, dal futuro del verbo volere sono teoricamente possibili, ma difficilmente reperibili ${ }^{9}$.

Oltre che dal verbo volere, appartenente alla categoria dei verbi modali in senso stretto, l'infinito passato può essere retto anche dal verbo preferire, sempre al condizionale, e, marginalmente, anche da desiderare, gradire ${ }^{10}$, ecc. $((7 d)$ e $(7 h))$, tutti appartenenti alla categoria dei verbi modali in senso lato.

(7) a. Preferisco laurearmi.

b. ?Preferisco essermi laureata ${ }^{11}$.

c. Preferirei laurearmi.

d. Preferirei essermi laureata.

e. Preferivo laurearmi.

f. ?Preferivo essermi laureata.

g. Avrei preferito laurearmi.

h. Avrei preferito essermi laureata.

Sia il verbo volere che il verbo preferire al condizionale seguiti da un infinito passato esprimono un desiderio relativo a un periodo anteriore al tempo della reggente. Più precisamente, come esemplificato da (8a)-(8d), i costrutti contenenti un infinito passato non negato servono a esprimere un desiderio relativo alle azioni anteriori non realizzate, mentre i costrutti contenenti un infinito passato negato esprimono un desiderio relativo alle azioni anteriori realizzzate ${ }^{12}$ (definibile anche come rimpianto). La possibilità di negare un infinito retto da volere ci porta alla conclusione che la

${ }^{9}$ Frasi come (6b) e (6f) sono considerate o inaccettabili o di dubbia accettabilità da parte dei parlanti madrelingua da noi consultati, per cui in questo contributo vengono contrassegnate con un punto di domanda. Il fatto che frasi simili nella realtà linguistica italiana siano caratterizzate da un uso (v. Skytte 1983: 110), ci pare, del tutto marginale, viene al momento lasciato da parte.

${ }^{10} \mathrm{Cfr}$. Desidererei/Gradirei/?Amerei/?Adorerei/?Odierei/?Detesterei essermi laureata prima.

${ }^{11}$ A differenza di ?Preferisco essermi laureata e ?Voglio essermi laureata, ritenute entrambe di dubbia accettabilità, frasi come Preferisco il fatto di essermi laureata (al fatto di aver trovato un lavoro) o Gradisco/Amo/Adoro/Odio/Detesto il fatto di essermi laureata sono del tutto accettabili. Secondo noi, *Voglio il fatto di essermi laureata e *Desidero il fatto di essermi laureata sono agrammaticali perché volere e desiderare esprimono volontà e/o desiderio, mentre i verbi preferire, gradire, amare, adorare, odiare, detestare esprimono gradimento, il che rende possibile il loro uso con la costruzione il fatto di + infinito passato, la quale indica un'azione realizzata.

${ }^{12}$ Negli esempi (8a)-(8d) volere e preferire sono sempre nella forma affermativa e l'infinito passato è quello che viene negato o meno. Sono comunque accettabili anche frasi come Non vorrei essermi laureata. 
costruzione di forma volere al condizionale + non + infinito passato non è una costruzione ristrutturata (cfr. Manzini et al. 1991: 514-516).

Dal momento che l'infinito serbo indica la contemporaneità o la posteriorità rispetto al tempo del verbo reggente, è chiaro che l'infinito passato italiano retto da volere o preferire al condizionale può essere tradotto in serbo soltanto con il costrutto di modo finito $d a+$ perfekt ${ }^{13}$. Quanto affermato è dimostrato dagli esempi (8a)-(8d), riportati di seguito insieme alle corrispondenti traduzioni in serbo:

(8) a. Vorrei essermi laureata prima.

(Volela bih/Želela bih da sam ranije diplomirala.)

b. Vorrei non essermi laureata così in fretta.

(Volela bih/Želela bih da nisam diplomirala tako brzo.)

c. Preferirei essermi laureata prima.

(Više bih volela da sam ranije diplomirala.)

d. Preferirei non essermi laureata così in fretta.

(Više bih volela da nisam diplomirala tako brzo.)

A questo proposito, riteniamo importante segnalare il fatto che nelle traduzioni serbe delle costruzioni italiane contenenti il verbo volere al condizionale seguito da un infinito passato non viene usato l'equivalente modale serbo hteti, bensì il verbo voleti 'amare' o, più raramente, želeti 'desiderare', sempre al condizionale (denominato potencijal in serbo) seguito dal costrutto di modo finito da + perfekt (v. (8a) e (8b)). Questo fatto ci fa concludere che il verbo serbo hteti, a differenza di volere e voleti (o želeti), non ammette, proprio a nessuna condizione, l'anteriorità del verbo retto.

Al fine di spiegare la preferenza della lingua serba per il condizionale di voleti (o želeti) piuttosto che per il condizionale di hteti come verbo reggente il costrutto $d a+$ perfekt (tenendo comunque presente il fatto che alla base di questa preferenza sta l'incompatibiltà di hteti con l'anteriorità del verbo retto), conviene sottoporre il costrutto serbo $d a+$ perfekt retto da voleti (o želeti) ad un esame più accurato. Dall'analisi degli esempi (8a) e (8b) emergono due fatti correlati fra loro:

1) i verbi voleti e želeti (entrambi appartenenti alla categoria dei modali in senso lato), potendo esprimere un desiderio relativo sia a un periodo contemporaneo (o posteriore) sia a un periodo anteriore, risultano essere lessicalmente più ricchi rispetto al verbo hteti (appartenente alla categoria dei modali in senso stretto), il quale esprime solo volizione relativa a un periodo contemporaneo (o posteriore). $\mathrm{Ci}$ pare questo il motivo per cui in

\footnotetext{
${ }^{13}$ L'infinito presente italiano retto da verbi modali corrisponde all'infinito (presente) serbo, commutabile con un costrutto di modo finito di forma $d a+$ prezent (congiunzione $d a+$ presente). Per più informazioni su questo costrutto v. Miškeljin (2012).
} 
serbo si ricorre all'uso di voleti (o želeti) al posto di hteti quando si vuole esprimere un desiderio relativo a un periodo anteriore. Il verbo serbo hteti, a causa della sua ristrettezza lessicale (esprime solo volontà), dispone dunque di una distribuzione più limitata rispetto al verbo italiano volere, il quale invece, esprimendo sia volizione che desiderio, pare essere usato molto più spesso del verbo desiderare ${ }^{14}$.

2) il costrutto $d a+$ perfekt retto da voleti (o želeti) al condizionale (cfr. le traduzioni delle frasi in (8a)-(8d)) pare molto simile alla subordinata condizionale di forma $d a+$ perfekt usata in serbo per indicare una condizione irreale passata. Le due costruzioni, usate entrambe in relazione a un verbo al condizionale nella reggente, non hanno lo stesso status all'interno del periodo. In (9a) il costrutto $d a+$ perfekt retto da voleti al condizionale è una subordinata completiva, mentre in $(9 \mathrm{~b}) d a+$ perfekt è una subordinata (non completiva) condizionale, detta anche protasi ${ }^{15}$.

(9) a. Volela bih da sam te pozvala u bioskop. (Vorrei averti invitato al cinema.)

b. Krenula bih s tobom na planinu da sam znala da ideš. (Sarei partita con te per la montagna se avessi saputo che ci andavi.)

Una somiglianza di questo genere è riscontrabile anche nelle corrispondenti frasi italiane. Per capirla meglio, bisogna fare un primo passo: usare al posto dell'infinito passato retto da volere (o preferire, ecc.) al condizionale (v. la traduzione italiana di (9a)) un costrutto di modo finito (al congiuntivo), come viene esemplificato da (10a), che pare dimostrare come la restrizione relativa all'impossibilità di usare un congiuntivo nella struttura subordinata in caso di coreferenza di soggetti non valga all'interno della costruzione costituita da volere al condizionale seguito da un congiuntivo con il soggetto coreferente con quello di volere. La stessa osservazione si riferisce a $(10 \mathrm{c})$, in cui al posto dell'infinito presente retto da volere al condizionale viene usato un congiuntivo imperfetto con il soggetto coreferente, nonché a preferire $((10 \mathrm{e})$ e $(10 \mathrm{~g}))$. Si tratta dunque di un'eccezione alla restrizione sopraccitata, che ci fa pensare che le costruzioni al congiuntivo retto dal condizionale di volere e preferire abbiano uno status particolare, dimostrato forse anche dal fatto che il condizionale, sia presente che passato, regge sempre un

${ }^{14}$ La ristrettezza lessicale di hteti sembra influire anche sull'alternanza di hteti e želeti in serbo quando reggono un SN (Hoću jastuk 'Voglio un cuscino' vs. Želim jastuk 'Desidero un cuscino'). Hteti $+\mathrm{SN}$ ci pare poco più frequente di želeti $+\mathrm{SN}$, mentre volere $+\mathrm{SN}$ sembra molto più frequente di desiderare $+\mathrm{SN}$.

${ }^{15}$ Per maggiori informazioni sulle frasi subordinate (completive e non) in serbo v. Ružić (2006a, 2006b) e Stanojčić \& Popović (2005). 
congiuntivo imperfetto per esprimere la contemporaneità e un congiuntivo trapassato per esprimere l'anteriorità. Questo status particolare pare basarsi sul fatto che anche in italiano, sebbene in protasi ci sia se e non che (10h), si intravede una certa analogia tra un periodo ipotetico contenente un verbo al condizionale (presente o passato) nella reggente (detta anche apodosi) e un congiuntivo (imperfetto o trapassato) nella subordinata condizionale, da una parte, e una costruzione costituita da volere o preferire al condizionale (presente o passato) seguito da un infinito (presente o passato), commutabile con un congiuntivo (imperfetto o trapassato), dall'altra. Anche le frasi di dubbia accettabilità esemplificate da (10i) e (101), in cui l'infinito passato (commutabile con un congiuntivo trapassato) non è retto da un condizionale passato, ma da un indicativo imperfetto, presentano una certa analogia con il periodo ipotetico in cui sia nella reggente che nella subordinata c'è un indicativo imperfetto $(10 \mathrm{~m})$.

(10) a. Vorrei che ti avessi invitato al cinema.

b. Vorrei essere più rilassata.

c. Vorrei che fossi più rilassata.

d. Preferirei essere più rilassata.

e. Preferirei che fossi più rilassata.

f. Preferirei essere stata più rilassata.

g. Preferirei che fossi stata più rilassata.

h. Sarei contenta se ti avessi invitato al cinema.

i. ?Volevo essermi laureata.

1. ?Preferivo essermi laureata.

m. Se volevo, mi laureavo.

\section{CONCLUSIONE}

In questo contributo abbiamo messo in risalto il fatto che, in italiano, tra tutti i verbi modali a controllo (sia quelli in senso stretto che quelli in senso lato), soltanto i verbi volere e preferire (marginalmente anche desiderare e gradire) possono reggere un infinito passato. Con la seguente restrizione però: questo uso risulta contrassegnato da una chiara preferenza della lingua italiana per il condizionale, presente o passato, dei verbi citati sopra. E infatti l'unico caso in cui un verbo modale a controllo può reggere una forma verbale indicante l'anteriorità. A questo proposito, abbiamo ritenuto necessario porre l'accento anche sul fatto che l'infinito passato italiano è commutabile con un costrutto di modo finito al congiuntivo trapassato anche se in caso di coreferenza di soggetti in italiano vale la restrizione sull'uso del congiuntivo nelle strutture subordinate rette da un verbo che richiede il congiuntivo. Abbiamo analizzato anche l'equivalente serbo della forma 
infinitivale composta italiana - il costrutto di modo finito $d a+$ perfekt, non commutabile con un infinito. Sia nel caso dell'infinito passato italiano che nel caso del costrutto $d a+$ perfekt abbiamo rilevato una certa analogia di modi e tempi rispettivamente con il periodo ipotetico italiano contenente un condizionale nella reggente e un congiuntivo nella subordinata e con il corrispondente periodo ipotetico serbo contenente un condizionale nella reggente e il costrutto $d a+$ perfekt nella subordinata.

\section{BIBLIOGRAFIA}

Benincà, P. (2003). Sintassi. In A. A. Sobrero (a cura di), Introduzione all'italiano contemporaneo: Le strutture (pp. 247-290). Roma - Bari: Editori Laterza.

Donati, C. (2008). Sintassi. Bologna: Il Mulino.

Gnjatović, T. \& Matasović, R. (2013). Some Observations on Verbs with Obligatory Control in Croatian. Rasprave, 39/2, 405-422.

Graffi, G. (1994). Sintassi. Bologna: Il Mulino.

Manzini, M. R., Salvi, G. \& Skytte, G. (1991). Frasi subordinate all'infinito. In L. Renzi \& G. Salvi (a cura di), Grande grammatica italiana di consultazione II (pp. 483-569). Bologna: Il Mulino.

Miškeljin, I. (2012). Infinitival complements in Serbian: A Generative Approach. Filolog, V, 199-209.

Moskovljević, J. (2008). Ogledi o glagolskoj potkategorizaciji. Beograd: Čigoja štampa.

Pranjković, I. (2013). Gramatička značenja. Zagreb: Matica hrvatska.

Ružić, V. (2006a). Dopunske rečenice u savremenom srpskom jeziku I. Zbornik Matice srpske za filologiju i lingvistiku, 49/1, 123-216.

Ružić, V. (2006b). Dopunske rečenice u savremenom srpskom jeziku II. Zbornik Matice srpske za filologiju i lingvistiku, 49/2, 105-266.

Salvi, G. \& Vanelli, L. (2004). Nuova grammatica italiana. Bologna: Il Mulino.

Skytte, G. (1983). La sintassi dell'infinito in italiano moderno. Copenaghen: Munksgaards Forlag.

Stanojčić, Ž. \& Popović, Lj. (2005). Gramatika srpskoga jezika. Beograd: Zavod za udžbenike i nastavna sredstva. 


\title{
ITALIAN PERFECT INFINITIVE EMBEDDED UNDER CONTROL MODALS AND ITS SERBIAN EQUIVALENT DA+PERFEKT
}

\begin{abstract}
Summary
The aim of this paper is to account for the distribution of the Italian perfect infinitive as a complement of control modals. We want to point out that among these verbs only volere and preferire (marginally desiderare e gradire) can have a verbal form indicating anteriority, i.e. a perfect infinitive as a complement, preferably if used in the present or past conditional. Our focus is also on the Italian perfect infinitive being commutable with a finite construction with a verb in the past perfect subjunctive although there is a restriction which says that in Italian, in case of subject coreference, it is not possible to use subjunctive mood in subordinate clauses embedded under a matrix verb requiring subjunctive. Our analysis is also related to the $d a+$ perfekt construction, which is the Serbian equivalent to the Italian perfect infinitive as a control modal verb complement. We also find that either the Italian perfect infinitive or the Serbian $d a+$ perfekt construction show a certain analogy concerning mood and time respectively with Italian conditional clauses containing a subjunctive verb and with Serbian conditional clauses containing the $d a+$ perfekt construction both related to a conditional matrix verb.
\end{abstract}

Keywords: Italian, Serbian, perfect infinitive, modal verbs, control, da + perfekt. 